\section{BRAZIULIAN JOURNAL \\ OF MEDICAL AND BIOLOGICAL RESHARCH}

www.bjournal.com.br
ISSN 0100-879X

Volume 43 (12) 1135-1244 December 2010

BIOMEDICAL SCIENCES

AND

CLINICAL INVESTIGATION

Braz J Med Biol Res, December 2010, Volume 43(12) 1160-1166

doi: 10.1590/S0100-879X2010007500128

A clinical gamma camera-based pinhole collimated system for high resolution small animal SPECT imaging

J. Mejia, O.Y. Galvis-Alonso, A.A. de Castro, J. Braga, J.P. Leite and M.V. Simões

The Brazilian Journal of Medical and Biological Research is partially financed by

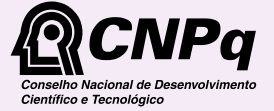

Ministério Conselho Nacional de Des
Cientifico e Tecnológico

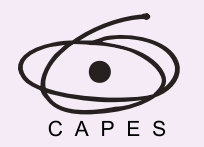

Ministério da Educação

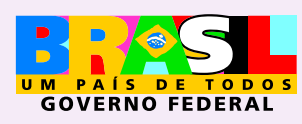

TFAPESP

Institutional Sponsors

Hotsite of proteomics metabolomics developped by:

$$
\frac{\mathrm{an}}{\mathrm{and}}
$$

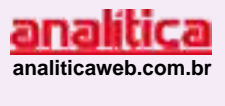

Therisio
Sc IEN T I I c 


\title{
A clinical gamma camera-based pinhole collimated system for high resolution small animal SPECT imaging
}

\author{
J. Mejia ${ }^{1}$, O.Y. Galvis-Alonso ${ }^{1}$, A.A. de Castro ${ }^{2}$, J. Braga ${ }^{4}$, \\ J.P. Leite ${ }^{3}$ and M.V. Simões ${ }^{2}$ \\ 1Departamento de Biologia Molecular, Faculdade de Medicina de São José do Rio Preto, \\ São José do Rio Preto, SP, Brasil \\ 2Departamento de Clínica Médica, ${ }^{3}$ Departamento de Neurociências e Ciências do Comportamento, \\ Faculdade de Medicina de Ribeirão Preto, Universidade de São Paulo, Ribeirão Preto, SP, Brasil \\ ${ }^{4}$ Divisão de Astrofísica, Instituto Nacional de Pesquisas Espaciais, São José dos Campos, SP, Brasil
}

\begin{abstract}
The main objective of the present study was to upgrade a clinical gamma camera to obtain high resolution tomographic images of small animal organs. The system is based on a clinical gamma camera to which we have adapted a special-purpose pinhole collimator and a device for positioning and rotating the target based on a computer-controlled step motor. We developed a software tool to reconstruct the target's three-dimensional distribution of emission from a set of planar projections, based on the maximum likelihood algorithm. We present details on the hardware and software implementation. We imaged phantoms and heart and kidneys of rats. When using pinhole collimators, the spatial resolution and sensitivity of the imaging system depend on parameters such as the detector-to-collimator and detector-to-target distances and pinhole diameter. In this study, we reached an object voxel size of $0.6 \mathrm{~mm}$ and spatial resolution better than 2.4 and $1.7 \mathrm{~mm}$ full width at half maximum when 1.5- and 1.0- $\mathrm{mm}$ diameter pinholes were used, respectively. Appropriate sensitivity to study the target of interest was attained in both cases. Additionally, we show that as few as 12 projections are sufficient to attain good quality reconstructions, a result that implies a significant reduction of acquisition time and opens the possibility for radiotracer dynamic studies. In conclusion, a high resolution single photon emission computed tomography (SPECT) system was developed using a commercial clinical gamma camera, allowing the acquisition of detailed volumetric images of small animal organs. This type of system has important implications for research areas such as Cardiology, Neurology or Oncology.
\end{abstract}

Key words: Pinhole collimator; SPECT; Small animal organs; Maximum likelihood; Instrumentation; Image processing

\section{Introduction}

In the last decades, and mainly since the development of knockout animals, small animals have become important tools in preclinical research, as models of the most different human diseases (1). In order to assess the effect of the experimental protocol, many procedures are based on the sacrifice of the animal to remove and process the organ or tissue of interest. On the other hand, imaging techniques allow in vivo sequential evaluation of functional and structural organ changes caused by the pathologic process or experimental protocol. On this basis, the same animal can be studied several times along the experiment and can be used as its own control. Therefore, in vivo imaging techniques are useful to reduce the duration of the experiment, diminishing costs and reducing ethical problems.

Single photon emission computed tomography (SPECT) is a functional imaging technique based on the emission of individual gamma ray photons from the organ of interest. An appropriate pharmaceutical for the specific organ is labeled with the gamma ray emitter (e.g., ${ }^{99 \mathrm{~m} T c}$ or ${ }^{123} \mathrm{I}$ ) and is then injected into the subject. After an appropriate time lapse, it becomes fixed to the tissue of interest as a marker of its functional or metabolic activity and planar images can be registered (2).

In clinical applications, planar images of the radiopharmaceutical distribution (also called projections) are obtained in most cases by using parallel collimators, in combination

Correspondence: J. Mejia, Departamento de Biologia Molecular, Faculdade de Medicina de São José do Rio Preto, Av. Brigadeiro Faria Lima, 5416, 15090-000 São José do Rio Preto, SP, Brasil. E-mail: mejia famerp@yahoo.com.br

Received February 10, 2010. Accepted November 3, 2010. Available online November 19, 2010. Published December $20,2010$. 
with a position-sensitive scintillation crystal, most commonly sodium iodide activated with thallium [ $\mathrm{Nal}(\mathrm{TI})]$. Fan beam and cone beam collimators can also be used for specific applications (3). A set of projections from different angles around the subject are registered. By means of appropriate computational manipulations, these projections are combined and a three-dimensional model of the emission distribution is obtained. When using parallel collimators, the most common procedure employed to reconstruct that three-dimensional model is the filtered back projection (FBP) based on the inverse radon transform (see, e.g., Ref. 4).

In linear dimensions, rodents are nearly one tenth the size of human beings. For this reason, clinical gamma cameras, as used in the clinical environment, are not appropriate for murine imaging and must be adapted, or special purpose instruments must be built. Single pinhole collimators are better suited for this application because of the associated intrinsic magnification factor. Depending on the ratio between the collimator-to-target and the collimatorto-detector distances (which gives the magnification), images are larger in size than the object. For this reason, spatial resolution becomes more dependent on the pinhole diameter than on the detector's intrinsic resolution, as can be seen in the expression:

$$
R_{o} \cong \sqrt{\left[\left(1+\frac{1}{M}\right) d_{\text {eff }}\right]^{2}+\left[\frac{R_{i}}{M}\right]^{2}} \quad \text { Equation 1 }
$$

where $R_{O}$ is the spatial resolution on the target, $M=d_{i} / d_{O}$ is the magnification factor, $d_{i}$ and $d_{0}$ are the distances from the collimator to the detector and to the target, respectively, $d_{\text {eff }}$ is the effective diameter of the pinhole, considering penetration of photons through its borders, and $R_{i}$ is the detector's intrinsic resolution.

However, there is a tradeoff between spatial resolution and sensitivity since, as the pinhole's diameter is reduced, the number of photons emitted by the target that actually reach the detector is reduced (5), according to the expression:

$S=\frac{d_{\text {eff }}^{2} \cos ^{3}(\theta)}{16 d_{o}^{2}}$

Equation 2

where $S$ is the sensitivity and $\theta$ is the angle between the symmetry axis of the imaging system and the line joining a radiation source and the pinhole $\left(d_{\text {eff }}\right.$ and $d_{O}$ have the same meaning as in Equation 1). Additionally, by increasing the magnification factor, the same quantity of photons is distributed on a larger area of the detector, reducing the number of photons per unit area.

The main objective of the present study was to upgrade a clinical gamma camera by developing a set of hardware and software tools in order to obtain high spatial resolution tomographic images of small animal organs. To acquire planar projections, we adapted a clinical gamma camera by building an appropriate shielding and pinhole collimator. Considering the heavy weight of the combined camera head and shielding as well as the target's size, the positioning errors introduced by rotating the detector around the target are assumed to hinder reaching the necessary image quality for organs of small animals. For this reason, we built a computer-controlled, step motor-based system that allows to precisely rotate the target and to maintain its position in front of the pinhole as the projections are registered. Finally, we implemented a computer code based on the maximum likelihood algorithm to obtain a three-dimensional model of the target's emission, from the set of projections registered through the pinhole collimator.

\section{Material and Methods}

\section{Hardware implementation}

We upgraded the hardware of a clinical gamma camera (DST/Sopha Medical Vision, USA) by building a set of items consisting of shielding, collimator insert and animal holder and positioning system. The shielding consists of a pyramidal acrylic structure covered with a 3-mm thick lead sheet. Considering the density $\left(11.34 \mathrm{~g} / \mathrm{cm}^{3}\right)$ and attenuation factor (the photoelectric absorption coefficient at 140 $\mathrm{keV}$ is $2.18 \mathrm{~cm}^{2} / \mathrm{g}$ ) of lead, this thickness is sufficient to guarantee a transparency of less than $0.1 \%$ at the $99 \mathrm{mTc}$ emission energy (6). The collimator insert corresponds to a 4-mm thick cylindrical piece of lead with a 1.5-mm diameter, 100-degree total acceptance angle, double knife pinhole drilled in it. In addition to being fitted with this insert, the shielding allows us to position larger squared inserts, to be used to test multipinhole and coded mask-based collimators (for a review on this application, see, e.g., Ref. 7). Finally, the target positioning system is based on a $50-\mathrm{mm}$ diameter PVC tube attached to a computer-controlled step motor, which allows us to precisely rotate and position the target, either phantoms or animals, in order to acquire the corresponding projections. Larger tubes can also be used depending on the animal's weight and size. A picture of the hardware development, installed on the detector of the DST/ Sopha gamma camera is shown in Figure 1. This structure is fixed to the gamma camera's detector by means of appropriate electromechanical connectors which, in spite of the weight of the system (nearly $30 \mathrm{~kg}$ ), allow its positioning with the target's tube along the vertical direction. This setup is important to obtain images of the heart of small animals, in order to minimize its displacement inside the thoracic cavity during the animal's rotation.

Special effort was devoted to mechanically guaranteeing an alignment between the different subsets of the system to better than $0.1 \mathrm{~mm}$, which certainly should not be an 
extreme challenge for appropriate precision machining tools.

\section{Software implementation}

In the dynamic mode, the proprietary gamma camera's software allows us to acquire a sequence of $128 \times 128$-element planar images without movement of the camera's gantry, in contrast to the SPECT mode, when the camera's head rotates around the target. A first computer code was implemented to synchronize the target's rotation with the registering timing of the gamma camera in the dynamic mode.

After recording, the projections are saved in DICOM format and transferred to a personal computer for processing. A second computer program was implemented to reconstruct the target's three-dimensional distribution of emission from the projections. The program corresponds to an implementation of the maximum likelihood algorithm (8), which consists of the iterative restoration procedure following the recursive expression:

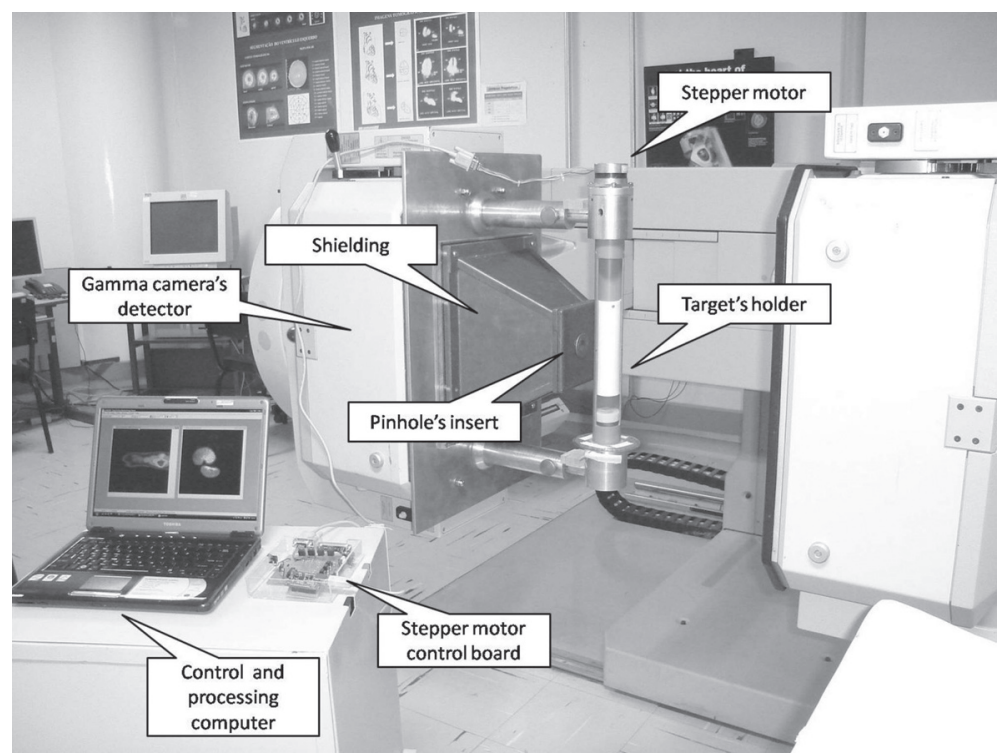

Figure 1. The system described in this report, with the main parts indicated, and attached to one of the two detectors of the DST dual-head clinical gamma camera. A parallel hole collimator was attached to the second detector in order to give assistance during the animal positioning procedure.
$I^{(l)}(i, j)=I^{(l-1)}(i, j) \frac{\left[\sum_{m, n} A(m, n ; i, j) \frac{R(m, n)}{\sum_{p, q} I^{(l-1)}(p, q) A(m, n ; p, q)}\right]}{\sum_{m, n} A(m, n ; i, j)}$

Equation 3

In this equation, I $(i, j)$ corresponds to the intensity distribution in the restored image, $R(m, n)$ corresponds to the intensity distribution in the recorded set of projections and $A(m, n ; i, j)$ represents the response of the instrument to a point source (also known as the Point Spread Function, or PSF). In this procedure, the starting value of the restored image, $I^{(0)}(i, j)$, is set to a uniform positive value, and the restrictive condition of $I^{(I)}(i, j) \geq 0$ is applied in every iteration.

The aforementioned algorithm was extended to three dimensions and considers the magnification effect of the pinhole collimator on the PSF. According to it, an initial model for the target's volumetric distribution of emission is proposed, as being a uniform one: $1^{0}(p, q, r)=1$. This emission model is projected through the instrument by means of the PSF to obtain a set of model projections, $P(m, n, o)=\sum I^{(l-1)}(p, q, r) A(m, n, o ; p, q, r)$. These model projections are compared to the recorded ones, resulting in a set of correction parameters, $R(m, n, o) / P(m, n, o)$, which are retro-projected to the object's space, again by means of the PSF. There, they are multiplied by the original target model to obtain an updated version of it, $I^{1}(p, q, r)$. The process is iteratively repeated until some finishing criterion is reached. Between individual iterations, a smoothing kernel was applied. In this procedure, the intensity of the volume element (or voxel) is calculated as the weighted mean of the $(3 \times 3 \times 3)$ neighborhood around the voxel, the weight being inversely proportional to the distance between the volume elements.

Even though this procedure is slower than the classic FBP algorithm, it produces better quality images in terms of signal-to-noise ratio and spatial resolution, and allows a better modeling of the physical process involved during image acquisition. The codes were implemented in C-Language by using the freeware compiler Dev-C++ (Bloodshed Software). For visualization of the final reconstructions, the freeware AMIDE Medical Image Data Examiner toolkit (9) was used.

\section{Data acquisition}

To test the feasibility of the imaging system, we acquired sets of projections from small size phantoms and in vivo rodents' organs. The phantoms consist of: 1) a 1-mm diameter catheter curved to form a three-armed target, with a $10-\mathrm{mm}$ distance between the arms; 2) a micro-Defrise phantom consisting of a pile of brass disks $10 \mathrm{~mm}$ in diameter and $3 \mathrm{~mm}$ in thickness, interspersed with activity-filled gaps of the same dimensions, and assembled inside the body of a $10-\mathrm{mL}$ syringe, and 3) a micro-Jaszczak phantom, composed of 4-, 3-, 2-, and 1-mm diameter hot rod sets, drilled in a cylindrical piece of aluminum. Pictures of the phantoms 
can be seen in Figure 2. Small quantities of $99 \mathrm{mTc}$ were used in the first two phantoms (less than $1 \mathrm{mCi}$ ) and $10 \mathrm{mCi}$ were used in the last one. Twenty equally spaced projections were acquired from the former two phantoms, while 40 equally spaced projections were acquired from the last one, on a 360-degree total angle. From them, the three-dimensional images were reconstructed as discussed previously. The axis of rotation was located $45 \mathrm{~mm}$ away from the pinhole and $295 \mathrm{~mm}$ away from the image plane. The catheter phantom was used to check the appropriate alignment of the mechanical system, as well as the values of the collimator-to-target and collimator-to-detector distances. To do so, its three-dimensional reconstruction was obtained under the assumption that the axis of rotation, the center of the pinhole collimator and the center of the detector were aligned. In the reconstruction software, we allow for the possibility of the pinhole and the axis of rotation to be out of alignment, in which case the correct positions are found by successive approximations and must be checked periodically.

We have also imaged the heart and kidneys of young $(200 \mathrm{~g})$ male Wistar rats. The animals were anesthetized with a ketamine/ xylazine combination and injected intravenously with $16 \mathrm{mCi} 99 \mathrm{mTc}-\mathrm{MIBI}$ and $10 \mathrm{mCi}$ 99mTc-DMSA, respectively. After $2 \mathrm{~h}$, the rats were again anesthetized and positioned inside the target tube. Thirty-six and 12, 30-s long, equally spaced projections were acquired for the imaging of heart and kidneys, respectively, on a 360-degree total angle. The axis of rotation was $45 \mathrm{~mm}$ away from the pinhole and $295 \mathrm{~mm}$ away from the image plane. All phantoms and animal projections were obtained on a $128 \times 128$-pixel matrix of $3.4 \mathrm{~mm} \times 3.4 \mathrm{~mm}$. Given the distances from the collimator to the axis of rotation and to the detector, those dimensions correspond to $0.61 \mathrm{~mm}$ in the middle plane of the object's space. A20\% energy window centered on 140 keV was considered during the acquisitions. Great care was taken to minimize animal suffering during the imaging procedures. All experimental procedures were performed according to the Faculdade de Medicina de São José do Rio Preto and Faculdade de Medicina de Ribeirão Preto, Universidade de São Paulo Institutional Guidelines for Animal Care and Use.
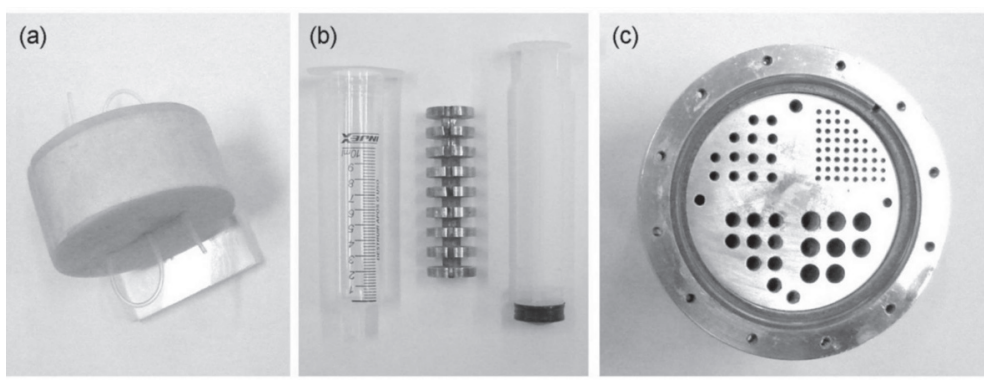

Figure 2. The phantoms used in the present study: a, 1-mm diameter, threearm curved catheter; $b, 10-\mathrm{mm}$ diameter, 3-mm thick emitting zone micro-Defrise phantom, and c, 4-, 3-, 2-, and 1-mm diameter hot rod micro-Jaszczak phantom.

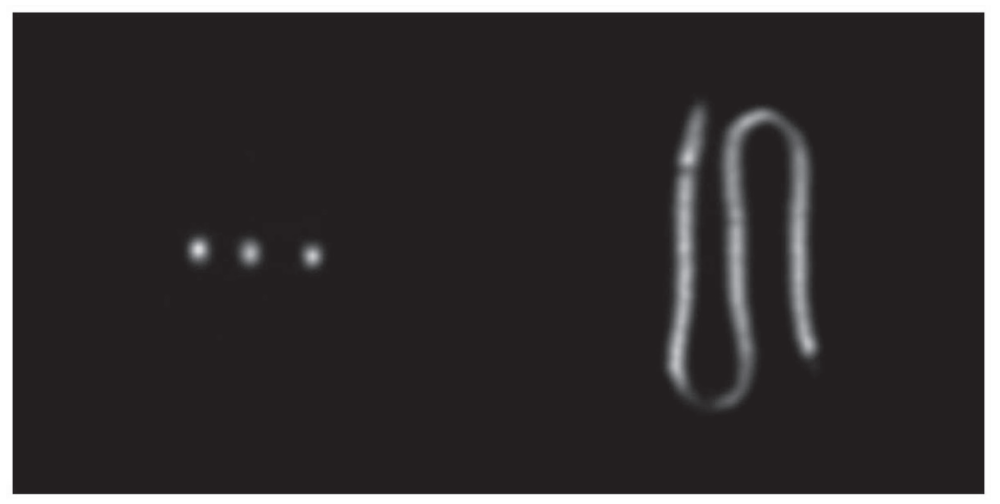

Figure 3. Vertical and horizontal intensity planes through the middle of the threedimensional reconstruction of the curved catheter phantom. The reconstruction was based on 20 projections acquired through a $1.5-\mathrm{mm}$ diameter pinhole collimator and produced with a maximum likelihood-based iterative decoding algorithm, after 10 iterations. A spatial resolution better than $2.4 \mathrm{~mm}$ was reached, determined as the mean full width at half maximum (FWHM) of the two-dimensional Gaussian functions fitted to the point-like profiles.

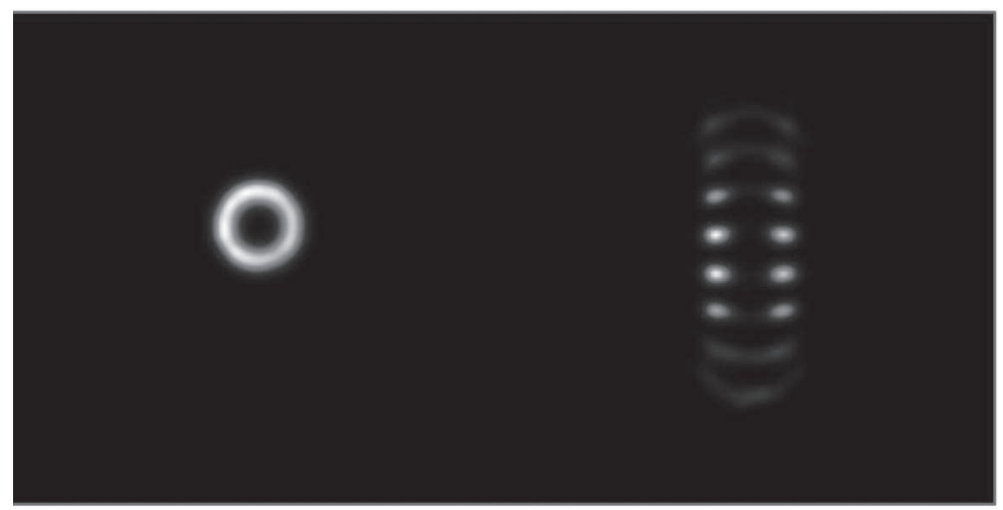

Figure 4. Vertical and horizontal planes through the middle of the three-dimensional reconstruction of the micro-Defrise phantom. The reconstruction was based on 20 projections acquired through a 1.5- $\mathrm{mm}$ diameter pinhole collimator and produced with a maximum likelihood-based iterative decoding algorithm, after 10 iterations. In the vertical plane (left), notice the clear identification of individual emitting disks and of the 6-mm diameter central axis of the phantom. Near the border of the field of view, the image becomes distorted, as can be seen in the horizontal plane (right), which is a consequence of the use of pinhole collimators. 


\section{Results}

In Figures 3 and 4 we show vertical and horizontal planes through the middle of the target and parallel to the rotation axis, from the reconstructed images of the curved catheter and micro-Defrise phantoms, after 10 iterations. In Figure 3 , notice that the individual arms of the catheter are clearly identified. There is no evidence of the star pattern associated with point-like sources, when the FBP algorithm is used for reconstruction. We determined the spatial resolution as the full width at half maximum (FWHM) of the point-like pattern in Figure 3, by adding the central 10 planes and fitting a two-dimensional Gaussian function. In doing so, we obtained a mean resolution of $2.4 \mathrm{~mm} \mathrm{FWHM}$, in clear accordance with the theoretical value for a $2.0-\mathrm{mm}$ diameter pinhole (effective diameter including partial transparency), a 5:1 ratio between detector-to-collimator and target-to-collimator distances, and a 4.0-mm detector's intrinsic resolution. Notice that, because of the finite size of the imaged object, the value determined for the spatial resolution is an upper limit, the real one being better than that shown here. By using a 1-mm diameter pinhole, a 1.7-mm FWHM spatial resolution was reached. In Figure 4, the individual emitting disks of the micro-Defrise phantom are clearly separated. Near the borders of the field of view (FOV), the image becomes distorted, a negative characteristic of the pinhole collimator (see, e.g., Ref. 10). Again, notice the absence of the starlike pattern associated with the FBP reconstruction method, the reduced background contribution and the clear identification of the central 6-mm diameter hole, corresponding to the axis of the phantom. In Figure 5, we present a vertical plane through the middle of the micro-Jaszczak phantom reconstruction, after 25 iterations. There, it can be seen that the hot rods of 2,3 , and $4 \mathrm{~mm}$ in diameter are appropriately resolved, again giving us an indication of the smaller objects that can be detected with this system, even in the presence of an intense background.

In Figure 6, we show the results of imaging the small animal's heart. We present the intensity distribution on 6 of the 36 acquired projections, together with the intensity distribution on planes parallel and perpendicular to the reconstructed heart's long axis, after 15 iterations. In Figure 7, we present, again, 6 of the 12 acquired projections, as well as a set of cuts through the three-dimensional reconstruction of the rat's kidneys, along the transaxial and horizontal directions, after 7 iterations. In both cases, rat's heart and kidneys, structure is easily recognizable in spite of the complexity of the target. Considering that these organs are roughly $10 \mathrm{~mm}$ in size, we can see that the developed system is appropriate for the proposed objective, even when few projections are used.
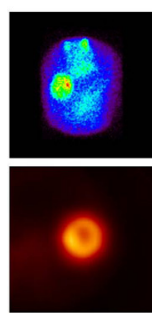

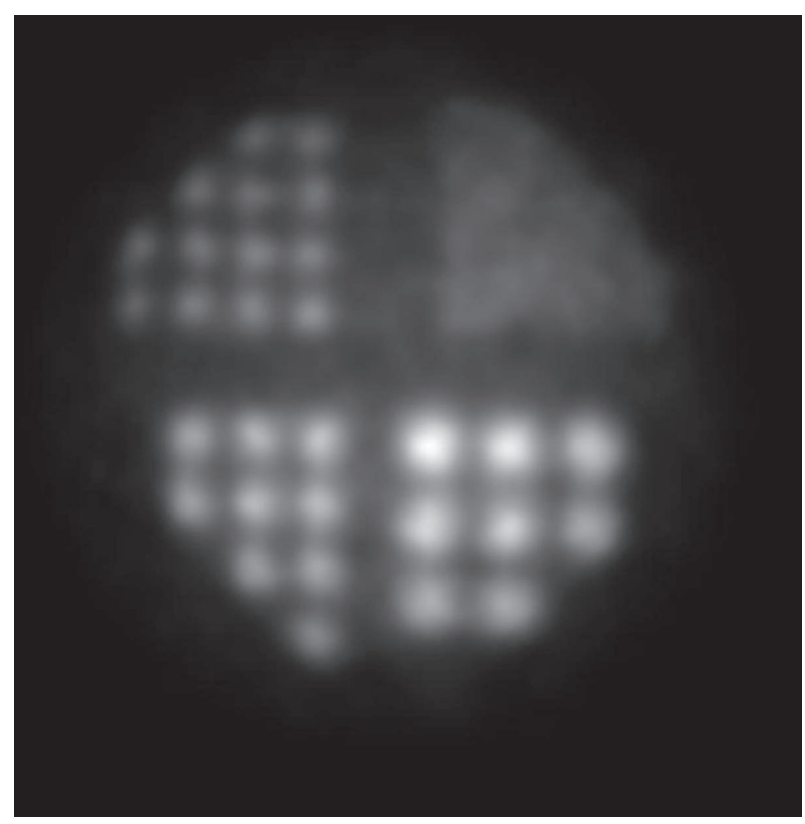

Figure 5. Vertical plane through the middle of the three-dimensional reconstruction of the micro-Jaszczak phantom after 25 iterations. Notice the clear identification of individual emitting rods from 2 to $4 \mathrm{~mm}$ in diameter. This result provides an estimate of the limiting size that can be resolved with the developed system.
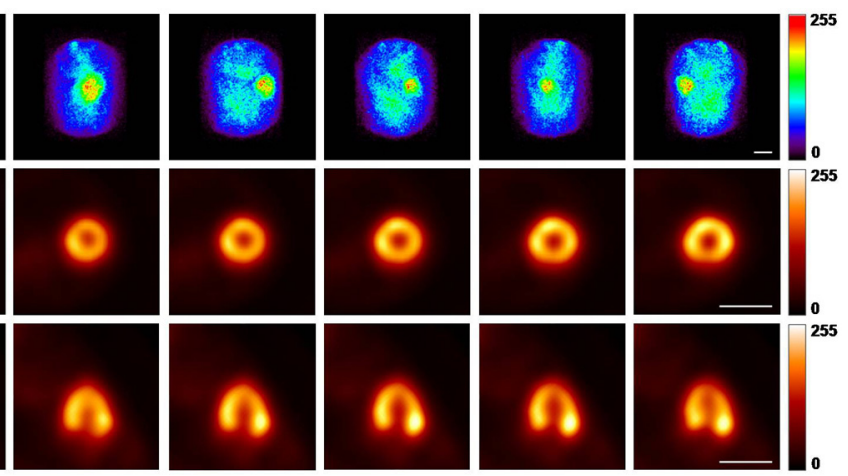

Figure 6. Planar projections and tomographic SPECT imaging of the rat's heart. Upper horizontal row: 6 of the 36 projections from the rat's heart. Middle and lower rows: transaxial planes through the long axis and horizontal planes parallel to the same axis of the three-dimensional reconstruction of the heart, after 15 iterations. A dose of $16 \mathrm{mCi}{ }^{99 \mathrm{~m} T c-M I B I}$ was injected into the tail vein, image recording was started $2 \mathrm{~h}$ after injection and each projection took $30 \mathrm{~s}$. The white horizontal bars correspond to $10 \mathrm{~mm}$.

\section{Discussion and Conclusions}

In the last decades, great effort has been dedicated to implementing strategies to appropriately image small volume targets, e.g., small animal organs. Special purpose instruments or adaptations of clinical gamma cameras have been proposed with this objective, mostly based on the use 

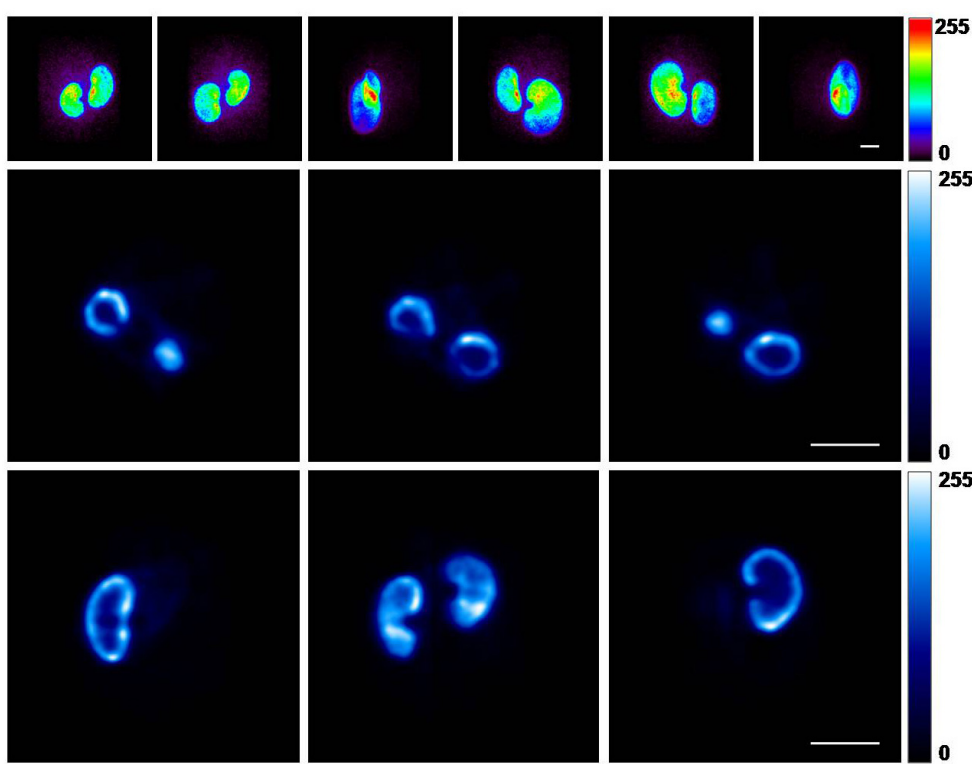

Figure 7. Planar projections and tomographic SPECT imaging of a rat's kidneys. Upper horizontal row: 6 of the 12 projections from the rat's kidneys. Middle and lower rows: sequence of planes through the three-dimensional reconstruction of the rat's kidneys, along the transaxial and vertical planes, after 7 iterations. A dose of $10 \mathrm{mCi}{ }^{99 m} \mathrm{Tc}-\mathrm{DMSA}$ was injected into the tail vein, image recording was started $2 \mathrm{~h}$ after injection and each projection took $30 \mathrm{~s}$. Notice the clear identification of the structure of the kidneys, namely the cortex, which is specifically labeled by DMSA. The white horizontal bars correspond to $10 \mathrm{~mm}$.

of pinhole-like collimators (see, e.g., Refs. 11-15). Most of the clinical imaging tests in Nuclear Medicine are performed with parallel collimators. For this reason, it is not common to find reconstruction algorithms for pinhole collimators implemented as part of the commercial instruments. Even in some gamma cameras, the pinhole collimator and corresponding shielding are not available.

In this study, we presented the results of the adaptation of a DST/Sopha clinical instrument to obtain high resolution images of small animal organs by building the appropriate hardware and software tools. With regard to the software, we implemented a three-dimensional version of the iterative image restoration algorithm known as the maximum likelihood algorithm. Compared with the classical FBP algorithm, it produces better quality decoded images and allows the inclusion of a more precise model of the instrument and of the physical phenomena involved in the image production process (16).

We tested our implementation by making images of small size phantoms and small animal organs. We show that, even in crowded volumes, small details of nearly 2 $\mathrm{mm}$ in diameter are easily recognizable, which is enough to obtain images of small animal organs (e.g., kidneys, heart and possibly brain) or tissues (e.g., tumors). The spatial resolution was determined as being better than $2.4 \mathrm{~mm}$ or $1.7 \mathrm{~mm}$ FWHM (depending on the pinhole diameter), by imaging sparse objects of $1 \mathrm{~mm}$ in diameter, in clear accordance with the theoretical value and the results reported by other groups $(11-13,17)$. Spatial resolution depends on several factors: detector's intrinsic resolution, collimator-to-detector distance, targetto-detector distance, and pinhole diameter. In this application, the first parameter is fixed. The ratio between the second and third ones, which gives the magnification factor, is determined by the size of the detector and the extension of the field of view. Given animals of almost the same size, the magnification factor is fixed, and care must be taken to avoid projecting the image of the target organ outside the detector as the animal rotates. Therefore, the only parameter we are free to modify is the pinhole's diameter. By diminishing the pinhole's size, spatial resolution is improved, even though sensitivity is also reduced (18-20). To compensate for this loss in sensitivity, some investigators have proposed to use multipinhole collimators. With this strategy, spatial resolution better than $1 \mathrm{~mm}$ FWHM has been reached with multiple pinholes smaller than $1 \mathrm{~mm}$, while maintaining appropriate sensitivity $(15,21)$. Alternatively, when imaging mouse organs, the magnification factor can be increased by diminishing the collimator-to-target distance, which has a positive effect on spatial resolution (21).

Alternatively, the penetrating ability of the gamma rays must be considered. In contrast to lower energy radiation, there is a probability of radiation coming through the collimator's border, causing the effective diameter to be greater than the physical one. By using radiopharmaceuticals labeled with substances emitting at lower energy, as is the case for ${ }^{125}$ (22), or materials with a higher absorption coefficient, as is the case for gold or platinum $(23,24)$, to build the inserts, it is possible to achieve further improvement of spatial resolution.

We also show that a small number of projections can be enough to obtain good quality reconstructions. In our study, we obtained good quality images of the rat's kidneys with as few as 12 projections. This is because of the reduced size of the imaged animals, in combination with the properties of the reconstruction algorithm, even though in most related reports a greater number of projections is acquired, from 64 to $120(11,13,25)$. With this characteristic, the necessary time for a study is greatly reduced, providing the opportunity to perform dynamic evaluation of the radiopharmaceutical biodistribution.

We are currently working on extending this work to include the use of multiple pinhole collimators in order to increase spatial resolution and sensitivity, which are mainly 
limited by the pinhole diameter, by making better use of the detector's sensitive area. As can be seen in Figure 6, when imaging specific organs, only a small area of the detector is used. By using a greater number of smaller pinholes ( 3 to 5), a more efficient use of the detector is achieved, improving sensitivity while at the same time maintaining spatial resolution. Additionally, the hole's diameter can be reduced to improve spatial resolution, and therefore we intend to use inserts of gold with 0.5 - to $1-\mathrm{mm}$ diameter holes.

Finally, it is noteworthy that the system described here involved a much reduced cost. Except for the gamma camera, the materials used here are all inexpensive and easy

\section{References}

1. Institute of Laboratory Animal Resources (U.S.). Committee on rodents, laboratory animal management: rodents. Washington: National Academy Press; 1996.

2. Cherry SR, Sorenson JA, Phelps ME. Physics in nuclear medicine. 3rd edn. Philadelphia: Elsevier Science; 2003.

3. Accorsi R. Brain single-photon emission CT physics principles. Am J Neuroradiol 2008; 29: 1247-1256.

4. Bruyant PP. Analytic and iterative reconstruction algorithms in SPECT. J Nucl Med 2002; 43: 1343-1358.

5. Beekman F, van der Have F. The pinhole: gateway to ultrahigh-resolution three-dimensional radionuclide imaging. Eur J Nucl Med Mol Imaging 2007; 34: 151-161.

6. XCOM. http://physics.nist.gov/cgi-bin/Xcom/xcom3_1. Accessed July 20, 2009.

7. Mejia J, Galvis-Alonso OY, Braga J, Correa R, Leite JP, Simoes MV. Methodological approaches to planar and volumetric scintigraphic imaging of small volume targets with high spatial resolution and sensitivity. Braz J Med Biol Res 2009; 42: 692-699.

8. Shepp LA, Vardi Y. Maximum likelihood reconstruction for emission tomography. IEEE Trans Med Imaging 1982; 1 : 113-122.

9. Loening AM, Gambhir SS. AMIDE: a free software tool for multimodality medical image analysis. Mol Imaging 2003; 2: 131-137.

10. Vanhove C, Defrise M, Lahoutte T, Bossuyt A. Three-pinhole collimator to improve axial spatial resolution and sensitivity in pinhole SPECT. Eur J Nucl Med Mol Imaging 2008; 35: 407-415.

11. Wu MC, Hasegawa BH, Dae MW. Performance evaluation of a pinhole SPECT system for myocardial perfusion imaging of mice. Med Phys 2002; 29: 2830-2839.

12. Acton PD, Kung HF. Small animal imaging with high resolution single photon emission tomography. Nucl Med Biol 2003; 30: 889-895.

13. Metzler SD, Jaszczak RJ, Patil NH, Vemulapalli S, Akabani G, Chin BB. Molecular imaging of small animals with a triplehead SPECT system using pinhole collimation. IEEE Trans Med Imaging 2005; 24: 853-862.

14. Franc BL, Acton PD, Mari C, Hasegawa BH. Small-animal SPECT and SPECT/CT: important tools for preclinical investigation. J Nucl Med 2008; 49: 1651-1663. to obtain locally. The most expensive product corresponds to the software development.

\section{Acknowledgments}

The authors wish to thank the technical staff of the Nuclear Medicine Section, HC-USPRP, mainly Márika Kristian de Oliveira, for invaluable help in preparing and manipulating the targets during the tests. J. Mejia is supported by FAPESP (\#07/50339-3 and \#07/58052-5) and J.P. Leite is supported by FAPESP CInAPCe (\#05/56447-7).

15. Rowland DJ, Cherry SR. Small-animal preclinical nuclear medicine instrumentation and methodology. Semin Nucl Med 2008; 38: 209-222.

16. Vandenberghe S, D'Asseler Y, Van de Walle R, Kauppinen $\mathrm{T}$, Koole M, Bouwens L, et al. Iterative reconstruction algorithms in nuclear medicine. Comput Med Imaging Graph 2001; 25: 105-111.

17. Quian J, Bradley EL, Majewski S, Popov V, Saha MS, Smith MF, et al. A Multi-Function Compact Small-Animal Imaging System Incorporating Multipinhole Standard and Helical SPECT and Parallel-Hole SPECT. IEEE Nucl Sci Symp Conf Rec 2006; M11-59: 2430-2438.

18. Acton PD, Choi SR, Plossl K, Kung HF. Quantification of dopamine transporters in the mouse brain using ultra-high resolution single-photon emission tomography. Eur J Nucl Med Mol Imaging 2002; 29: 691-698.

19. Difilippo FP. Design and performance of a multi-pinhole collimation device for small animal imaging with clinical SPECT and SPECT-CT scanners. Phys Med Biol 2008; 53: 41854201.

20. Quian J, Bradley EL, Majewski S, Popov V, Saha MS, Smith MF, et al. A small-animal imaging system capable of multipinhole circular/helical SPECT and parallel-hole SPECT. Nucl Instrum Methods Phys Res A 2008; 594: 102-110.

21. Goertzen AL, Jones DW, Seidel J, Li K, Green MV. First results from the high-resolution mouseSPECT annular scintillation camera. IEEE Trans Med Imaging 2005; 24 : 863-867.

22. Beekman FJ, McElroy DP, Berger F, Gambhir SS, Hoffman EJ, Cherry SR. Towards in vivo nuclear microscopy: iodine-125 imaging in mice using micro-pinholes. Eur J Nucl Med Mol Imaging 2002; 29: 933-938.

23. Cusanno F, Accorsi R, Cinti MN, Colillia S, Fortunad A, Garibaldi F. Molecular imaging by single-photon emission. Nucl Instrum Methods Phys Res A 2004; 527: 140-144.

24. Tenney CR. Optimizing gold and platinum pinhole collimators for imaging of small volumes at ultra-high resolution. IEEE Nucl Sci Symp Conf Rec 2001; 3: 1597-1599.

25. Cao Z, Bal G, Accorsi R, Acton PD. Optimal number of pinholes in multi-pinhole SPECT for mouse brain imaging - a simulation study. Phys Med Biol 2005; 50: 4609-4624. 